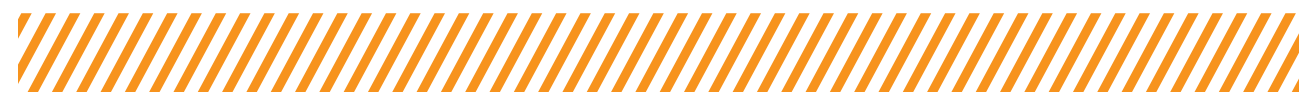

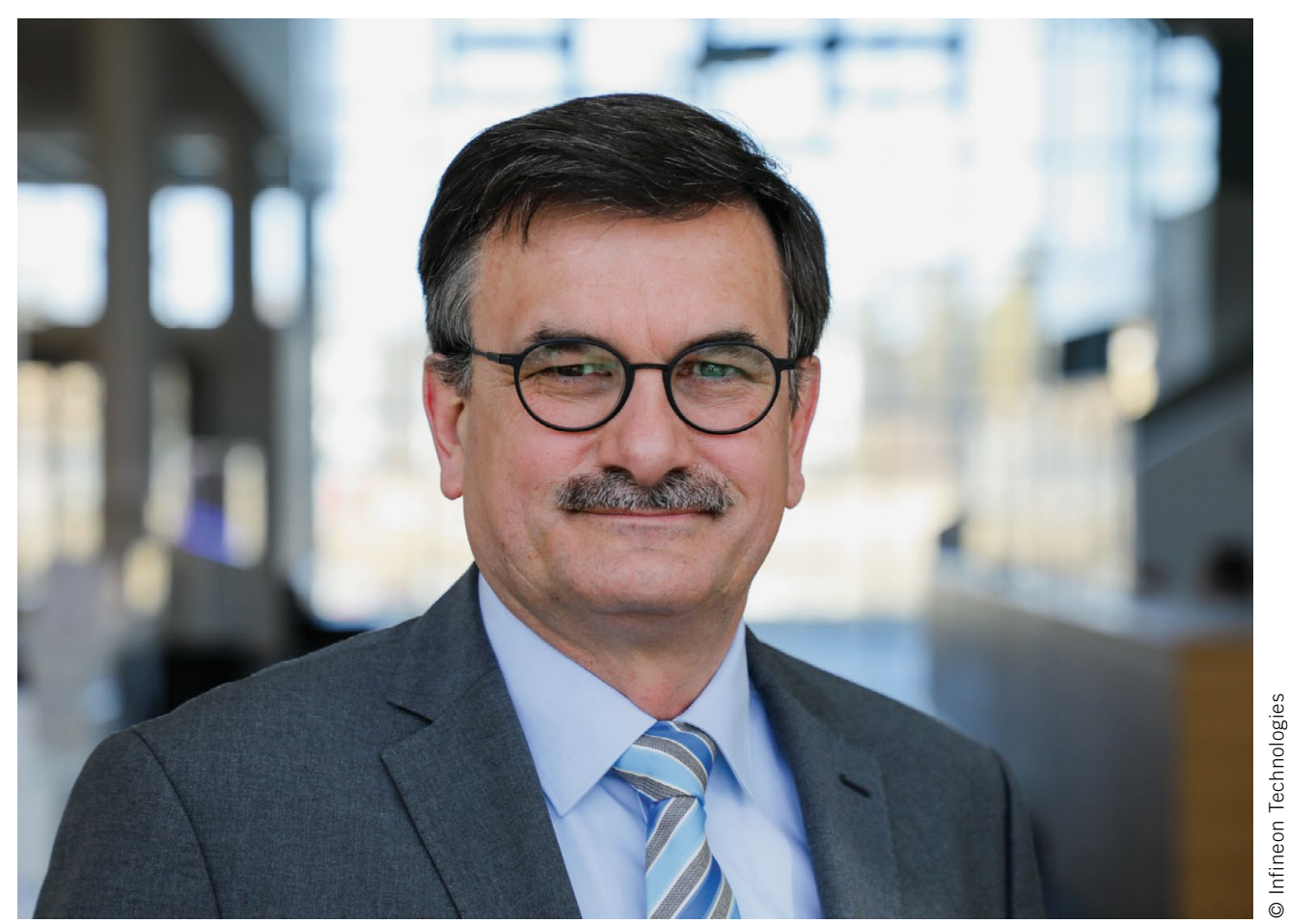

Hans Adlkofer

Senior Vice President Automotive of Infineon Technologies in Neubiberg (Germany)

\section{Safety and Security Need Hardware}

As a consequence of the coronavirus pandemic, 2020 saw a decline in the number of road fatalities in Germany by 322 : That is the good news. But let us not delude ourselves, we are still far away from the goal of Vision Zero as declared by the Traffic Club Germany (VCD) in 2009. Despite seat belts, airbags, ABS, and new assistants such as the emergency brake assistant, the number of traffic deaths has remained at a high level over the past four to five years. The number has even increased slightly in several countries. The 2724 deaths in Germany alone are 2724 too many. Which is why achieving the goal of Vision Zero means that we need both more safety and, to be precise, more security. The fact that personal individual mobility is on the brink of a revolution helps us. We should take advantage of this because the goal of Vision Zero is attainable with the introduction of novel Advanced Driver Assistance Systems (ADAS) and with the prospect of Autonomous Driving (AD).

Sensors are significant components necessary for ADAS and AD. For decades now, we have relied upon a range of sensor solutions in personal and commercial vehicles such as radar and, sporadically, lidar systems. Just recently, an electro-mechanical microsystem (MEMS) was launched for microphones that can be used on vehicle exteriors. Microphones as extensions to our senses are no longer limited to forming the basis of speech assistants that make the life of a driver simpler. They are also now capable of improving safety as first-responder vehicles such as ambulances or fire trucks can be perceived much earlier.

The semiconductor industry is continuing to develop new hardware-based solutions that grace the vehicle with additional senses and make them safer. And this is indeed necessary in view of the increasing complexity of road traffic due to the heavy use of traffic routes by a range of very different participants. We need sensors that support the vehicle so that external events and situations can be recognized unambiguously, and suitable actions taken to prevent an accident.

In addition, vehicles must be securely connected with the infrastructure and other vehicles to enable the early recognition of dangerous situations. This would result not only in the recognition of first-responders in close proximity but also include information regarding black ice or critical driving situations such as an emergency braking event; dangers that are not necessarily within a classical sensor's scope of perception. Connectivity presupposes data security under the motto "no safety without security." Compromised data, irrespective of whether they originated from a consciously executed attack or an incorrectly downloaded update, represent a security threat.

I am very confident that the goal of Vision Zero can be reached with a sensible combination of sensors in the overall vehicle system and protection via security chips. Based on hardware. 